\title{
A microfabricated physical sensor for atmospheric mercury monitoring
}

\author{
B. Mazzolai*, V. Mattoli, V. Raffa, G. Tripoli, D. Accoto, A. Menciassi, P. Dario \\ a Scuola Superiore Sant'Anna, P.zza Martiri della Libertà 33, 56127 Pisa, Italy
}

Received 10 June 2003; received in revised form 1 January 2004; accepted 12 January 2004

Available online 5 March 2004

\begin{abstract}
A new microfabricated physical sensor for elemental gaseous mercury $\left(\mathrm{Hg}^{0}\right)$ determinations has been developed and experimentally tested by the authors. $\mathrm{Hg}^{0}$ represents $90-99 \%$ of atmospheric mercury forms. The sensor is based on the technique of resistivity variation of thin gold film, characterised by high selectivity and absence of optical parts. The sensor consists of four identical thin gold film resistors mounted in Wheatstone bridge configuration. Two resistors work as sensitive elements and the others as reference, in order to minimise the influence of temperature variation. The absorption of $\mathrm{Hg}^{0}$ on the gold film produces a change in the resistivity of the amalgam. Far from the saturation, this change is proportional to the amount of the absorbed $\mathrm{Hg}^{0}$. The adsorption behaviour of the sensor deposited by sputtering on two different substrates (glass and Printed Circuit Board (PCB)) have been investigated. The sensors showed to work in a large range of linearity and need a low power during the regeneration process. Sensors on glass and PCB substrates underwent numerous regeneration cycles without inflicting any mechanical or electrical damages to the resistors. The presented experimental results describe the features of both sensors pointing out advantages and drawbacks of the used substrates. The PCB substrate seems to have more suitable characteristics for developing a new mercury 'smart' sensor.
\end{abstract}

(C) 2004 Elsevier B.V. All rights reserved.

Keywords: Mercury; Sensor; Gold; Thin film; Absorption

\section{Introduction}

Mercury represents one of the main environmental pollutants of our planet. A significant amount of research indicates that natural and anthropogenic sources can redistribute this element in the atmosphere, soil and water systems through a complex combination of transport and transformation. Studies on risk assessment and effects caused by mercury on human health, drive the scientists and decision makers to consider this metal as a global pollutant [1-3]. The work of the Authors has, as final goal, the development of a low cost mini-analyser able to work in cooperative way, thus forming monitoring networks, which produce an enhanced knowledge of the dispersion of a pollutant, and which can be ultimately integrated in the health-oriented monitoring system. In particular, in the present work, a new microfabricated sensor based on the resistivity variation of thin gold film, is described. This sensor is designed to measure elemental gaseous mercury $\left(\mathrm{Hg}^{0}\right)$ concentrations, which represents 90 to $99 \%$ of atmospheric mercury forms [3].

\footnotetext{
* Corresponding author. Tel.: +39-050-883414; fax: +39-050-883402. E-mail address: b.mazzolai@mail-arts.sssup.it (B. Mazzolai).
}

The described work is carried out within the on-going EU-project EMECAP (European Mercury Emissions from Chlor-Alkali Plants; Contract No. QLK4-CT-2000-00489).

\section{Design}

\subsection{Resistivity variation working principle}

The gaseous mercury sensor presented in this paper is based on the resistivity changes of thin gold films. The sensor consists of four identical thin gold film resistors mounted in the Wheatstone bridge configuration. In order to minimise the influence of temperature variation, two resistors work as sensitive elements while the others work as reference. The absorption of mercury on the gold film produces a change in the resistivity of the film itself. The resistance increase, due to a partial mercury overlay on gold, is produced by the change in the surface scattering of the electrons [4-7]. Far from the saturation, this change is proportional to the amount of mercury absorbed. After calibration, the signal outgoing from the Wheatstone bridge can be directly related to the concentration of mercury in air. For high mercury concentration in air, the resistance of the sensitive 
elements approaches the saturation, i.e. the variation of resistivity becomes asymptotically zero. In order to reuse a saturated mercury sensor, a regeneration step is required. To move the equilibrium versus the desorption process a direct heating of gold resistors over $100^{\circ} \mathrm{C}$ is applied.

\subsection{Fabrication}

The deposition of thin gold films is performed by sputtering deposition. The used sputtering machine is a radio frequency model (Sputtering Sistec, model DCC 150) operating at a constant pressure of $1 \mathrm{~Pa}$, using a gold target (purity 99.999\%). Pure Argon (purity 99.999\%) was used as sputtering gas. The thickness of the gold film $(200 \mathrm{~nm})$ was measured using a sputtering crystal sensor and the film surface morphology of the substrates was studied with Atomic Force Microscopy (AFM). After deposition, the sensor was annealed for $2 \mathrm{~h}$ at $130^{\circ} \mathrm{C}$ to release the intrinsic stress due to the thermal mismatch. Particular attention has been paid to the choice of the substrate. Since the sensor is designed to operate in a cheap and miniaturised device, the substrate must assure quality of adhesion as well as a low cost and possibility of batch fabrication. The four resistances have been deposited onto two types of substrate, thus fabricating two series of sensors. The first sensors' generation has been realised on glass (thickness $=0.1 \mathrm{~mm}$ ), with a superficial roughness of about $80 \mathrm{~nm}$, while the second one on Printed Circuit Board (PCB) (thickness $=1.6 \mathrm{~mm}$ ), characterised by a superficial roughness of about $300 \mathrm{~nm}$. The overall dimensions of the glass and PCB sensors are $12 \mathrm{~mm} \times$ $12 \mathrm{~mm} \times 0.10 \mathrm{~mm}$ and $29.35 \mathrm{~mm} \times 20.80 \mathrm{~mm} \times 1.60 \mathrm{~mm}$, respectively. The main advantages of using glass as substrate for developing the mercury sensor are its high thermal stability, surface smoothness and low cost, while PCB offers the possibility to easily obtain the electronic contacts and integrate the conditioning electronics on board.

\section{Experimental results}

\subsection{Experimental set-up}

The set-up used in the experimental activities is described. The fluidic circuit includes an air filter, a chamber containing the mercury sensor, and an air pump (KNF, NMP 05M; $0.51 \mathrm{~min}^{-1}, 500 \mathrm{mbar}$ in aspiration). The circuit is in aspiration to reduce the pollution of mechanical components continuously exposed to air. The components are connected by silicon tubes. A National Instruments DAQ Card (model 6024E) and a dedicate software was used to control the excitation parameters of the sensor and to acquire the experimental data. A $500 \mu \mathrm{l}$ sample of mercury saturated air is sucked from a thermos kept at controlled temperature and injected into the aspiration air using a precision manual syringe. A sampling flow rate $0.51 \mathrm{~min}^{-1}$ and a sampling time of $30 \mathrm{~s}$ were used. The readout was made after $30 \mathrm{~s}$ from

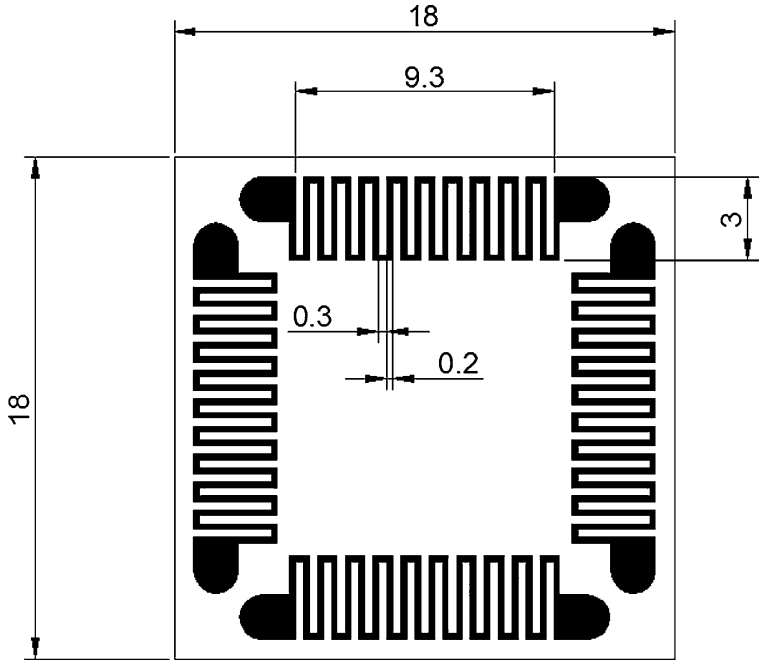

Fig. 1. Footprint of the four resistances linked in a Wheatstone bridge configuration. All dimensions are in millimeters. The thickness and exposed surface of gold film are about $200 \mathrm{~nm}$ and $25.4 \mathrm{~mm}^{2}$, respectively.

the turn-off of the pump in order to allow the recovery of thermal equilibrium, perturbed by massive air flow rate.

\subsection{The first version of the sensor}

The footprint of the first version of the sensors on both PCB and glass substrates is represented in Fig. 1. The sensor presents an exposed surface of about $25.4 \mathrm{~mm}^{2}$. Experiments have been carried out to test the linearity of the sensors. Fig. 2 shows the results of a comparative experiment carried out on a glass and a PCB sensor. The outcome tension from the Wheatstone bridge was plotted as a function of the quantity of mercury injected into the fluidic circuit. The experimental data and the relative graph obtained show a high readout variation and a manifestly linear trend.

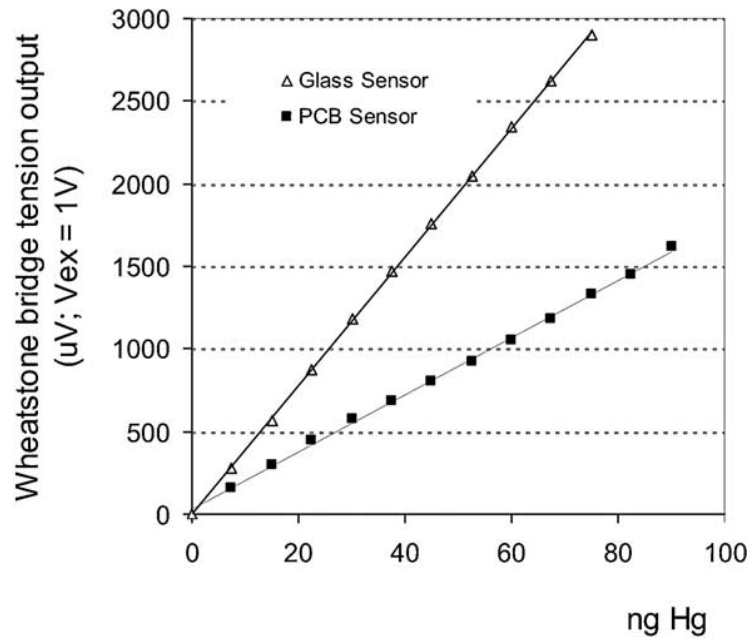

Fig. 2. Signals obtained from glass and a PCB sensors plotted as a function of the amount of mercury saturated air injected in air circuit. The potential used for the Wheatstone bridge excitation is $1 \mathrm{~V}$. The average resistance is for both sensors about $400 \Omega$. 
The average sensitivity of the glass and PCB sensors is about $48 \mu \mathrm{VV}_{\mathrm{ex}}^{-1}$ and $20 \mu \mathrm{VV}_{\mathrm{ex}}^{-1} \mathrm{ng}^{-1}$ of injected mercury, respectively. On the basis of these results, the detection limit can be estimate around $100 \mathrm{pg}$ of mercury. The change in sensitivity between glass and PCB sensors is related to the different film morphology. In particular, the film response to mercury depends strongly on the film properties such as surface defects, crystallite structure, grain boundaries and film thickness; in the process of physical vapour deposition of a thin film these proprieties are closely related to the substrate morphology [8]. A dedicated series of comparative saturation test was also performed in order to assess the linear response of sensors and their saturation limits. In these tests, an increased amount of mercury saturated air was injected in the air circuit until saturation. The experiment results showed that the sensor linear response limit (similar in both sensors) is around $300 \mathrm{ng}$ of injected mercury and the saturation limit is around 5000 ng of injected mercury.

\subsection{The regeneration}

Different techniques can be used to heat the resistors. In order to reduce the power consumption, the most efficient way is to use Joule effect self-heating, by short-circuiting the two passive resistances and supplying a regeneration voltage to the bridge.

Some tests were carried out in order to evaluate the 'sensor endurance' to regeneration, i.e. the minimum number of regeneration cycles which can be performed without inflicting any mechanical or electrical damage to the resistors. Unused sensors on glass and PCB substrates underwent numerous regeneration cycles after each of which the resistance variation was measured. After more than 1300 regeneration cycles no damage was observed. In order to investigate the behaviour of glass and PCB sensors during the regeneration process, the sensors were contaminated by mercury until to achieve the non linear adsorption limit area. During the test phase, only one of the four resistances was regenerated and the sensor temperature was monitored by a $k$-thermocouple (15 $\mu \mathrm{m}$ diameter) located on the film surface. The experimental results are showed in Fig. 3: a resistance temperature above $100^{\circ} \mathrm{C}$ is necessary to obtain a significant sensor regeneration. In Fig. 4 the curves "glass" and "PCB" report, respectively the temperature obtained at different regeneration currents for the two types of sensors. The PCB sensor requires a higher regeneration current to obtain the same temperature used for the glass sensor, because of its higher thermal inertia. To solve this problem a thinner PCB board was used and a new gold film design with a small exposed surface was fabricated and tested. The results of the mentioned design and microfabrication changes are represented by the "new PCB" curve in Fig. 4. As suggested by experimental and simulation results, this new sensor is characterised by a lower power consumption respect to the glass sensor.

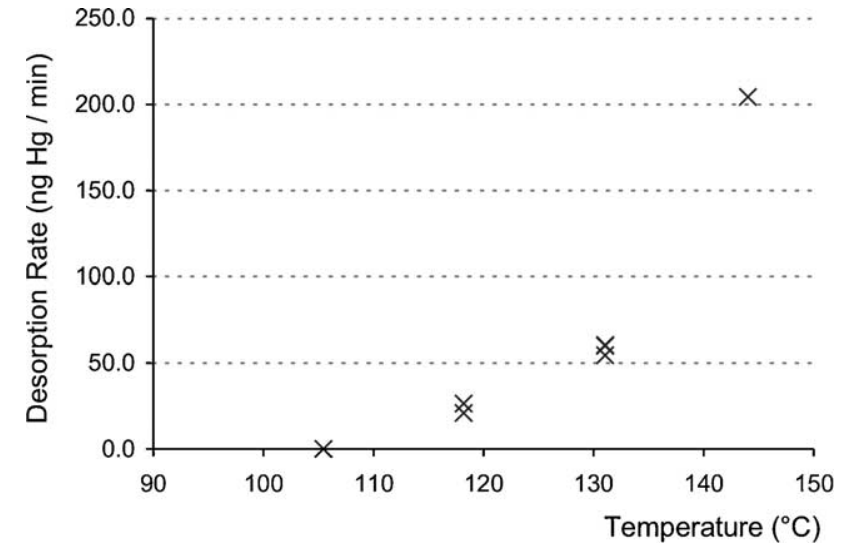

Fig. 3. Desorption rate of mercury for saturated gold films as a function of regeneration temperature.

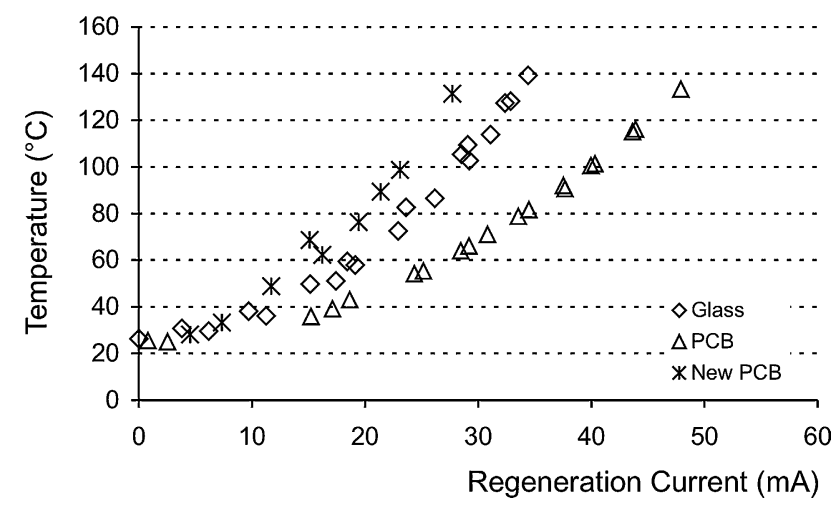

Fig. 4. Temperature measured on regenerated resistors as a function of current applied to the bridge for the fist version of sensor (glass and PCB substrates) and for the new version.

\subsection{The last version}

Fig. 5 reports the footprint of the PCB new sensor. The substrate thickness has been reduced to $0.8 \mathrm{~mm}$ in order to decrease the thermal inertia of the support. Furthermore,

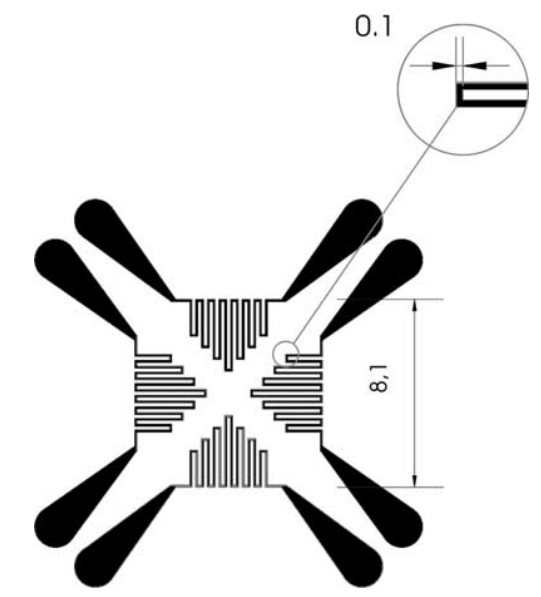

Fig. 5. Footprint of "new PCB" sensor. All dimensions are in millimeters. The thickness and exposed surface of gold film are about $200 \mathrm{~nm}$ thick and $6.4 \mathrm{~mm}^{2}$, respectively. 


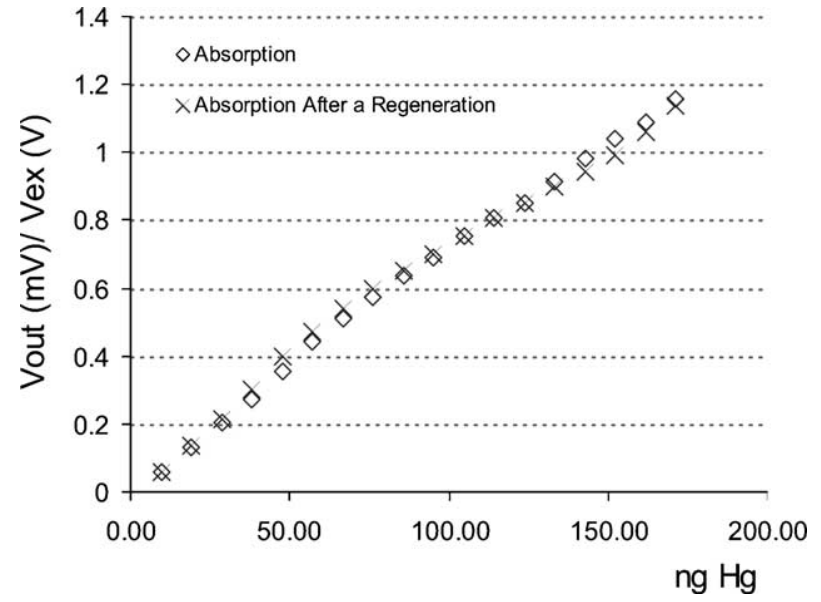

Fig. 6. Atomic mercury adsorption in the "new PCB" sensor before and after a regeneration cycles. The average resistance is about $320 \Omega$.

the gold exposed adsorption surface has been quartered to reduce the thermal dissipation and the power consumption.

Some trials were performed to test the behaviour of the new sensor, in particular to confirm the linear response and evaluate the saturation limit. In Figs. 6 and 7 the outcome tension from the Wheatstone bridge was plotted as a function of the quantity of mercury injected into the fluidic circuit. In order to evaluate whether reiterated regeneration steps can perturb the capacity of the gold film to trap mercury, absorption measurements were performed after the sensor regeneration. The results of two trials performed on the PCB sensor in the same operative conditions before and after a regeneration process (see Fig. 6) demonstrate that no change occurs in sensor adsorption capacity. The average sensitivity of the new sensor is about $7 \mu \mathrm{V} \mathrm{V}_{\text {ex }}^{-1}$. Compared to the old sensors, the new sensor showed a bigger linear response limit (about $800 \mathrm{ng}$ of mercury injected) and a comparable saturation limit (see Fig. 7). The sensor behaviour change in the range of linear adsorption compared to the old sensor on the same substrate is related to the more drastic thermal annealing on working sensors due to reduction of thermal resistances. The thermal stress has the effect

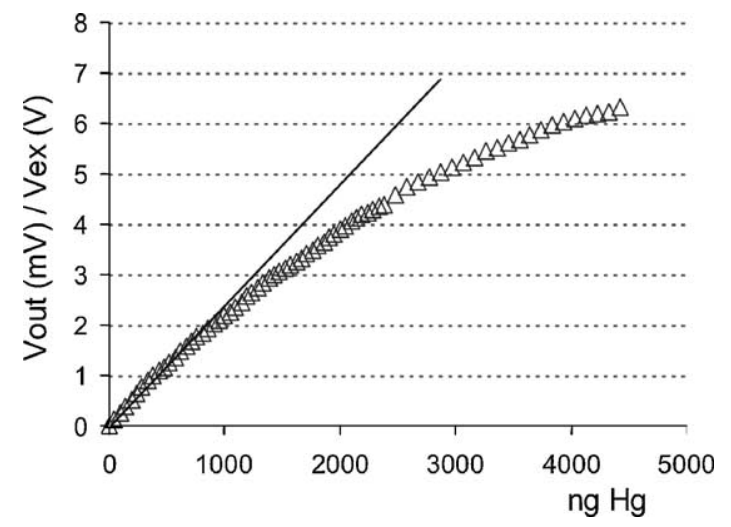

Fig. 7. Saturation curve. The test was performed on the same sensor of Fig. 6. to produce a more stable film morphology with a higher degree of recrystallization and a consequent loss in sensitivity.

\subsection{The chemical-physic behaviour of the sensor}

The comprehension of the chemical-physic mechanisms related to the mercury collection and adsorption at the gold interface and the factors affecting the efficiency is crucial for obtaining significant and reliable results from experimental tests. Data reported in literature [8] shows that for thin gold film exposed to gaseous metallic mercury the adsorption rate is dependent on the mercury concentration in the air flow. This behaviour can be explained considering two different processes involved in the mercury gold interaction: (1) mass transport of mercury at the deposition zone from the bulk gas; (2) adsorption and reaction of mercury on the interface. The transport in the gas phase takes place through diffusion, proportional to the diffusivity of the gas and the concentration gradient across the boundary layer (Fick's first law):

$N_{\mathrm{Hg}}:=\delta \frac{\mathrm{d} c_{\mathrm{Hg}}}{\mathrm{d} x}$

where $N_{\mathrm{Hg}}$ is the mercury flow rate, $\delta$ the diffusivity of the gas and $c_{\mathrm{Hg}}$ is the mercury concentration in the air flow. The temperature and mercury concentration regulate the absorption rate at the interface. The mathematical relation can be expressed by Langmuir isotherm equation (type I):

$Q_{\mathrm{Hg}}:=Q_{\mathrm{sat}} \frac{\alpha c_{\mathrm{sat}}}{1+\alpha c_{\mathrm{sat}}}$

where $Q_{\mathrm{Hg}}$ is the mercury amount on solid phase, $Q_{\text {sat }}$ the mercury amount on solid phase at saturation, $c_{\text {sat }}$ the concentration of saturated mercury vapour and $\alpha$ is a parameter. For low mercury concentration in the air flow, the absorption process proceed faster than the rate at which mercury is supplied at the interface by diffusion: the process is limited by diffusion and an increase of the film resistance proportional to the mercury concentration is expected. For high mercury concentration in the air flow, the rate-limiting step becomes the surface absorption: mercury supplied at the surface by diffusion cannot be consumed with a resulting flatting of the gradient concentration at the boundary layer. In the latter case, the film resistance variation becomes independent from the mercury concentration just depending from the absorption capability of the sensor. This explains the behaviour observed in Fig. 8 which reports the sensor response to the mercury concentration in the air flow. The suitable mercury concentration in the flow air was obtained through injection of a known amount of saturated vapour mercury into a constant volume of sucked air in the fluidic circuit. The experimental results show a sensor response proportional to the mercury concentration for low values and independent for high ones. 


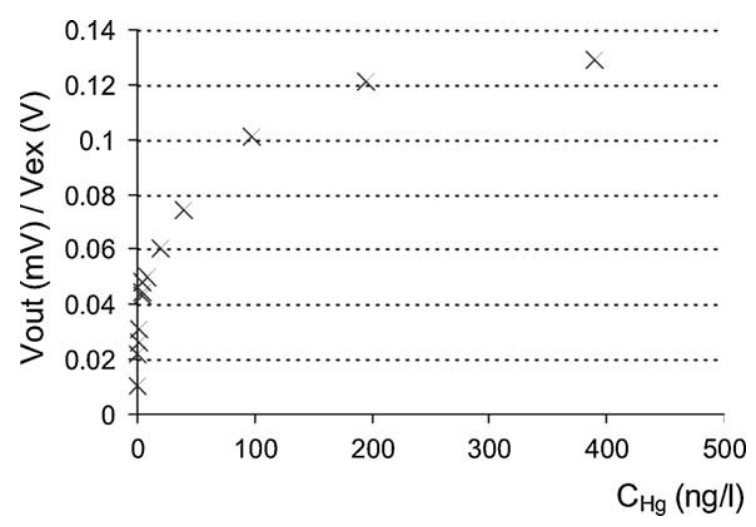

Fig. 8. Sensor response as a function of the mercury concentration in the air flow. Each experimental point refers to the same volume $(0.51)$ of sucked air in the fluidic circuit. The test was performed on the same sensor of Figs. 6 and 7.

\section{Conclusion}

Microfabricated sensors for atmospheric mercury determinations have been described and experimentally tested. The sensors are based on the resistivity variation of thin gold film technique. Experiments on the linearity of the sensors in function of mercury adsorption on two different substrates (glass and PCB) have been carried out. The sensors work in a large range of linearity and need low power during the regeneration process. Sensors on glass and PCB substrates underwent numerous regeneration cycles without inflicting any mechanical or electrical damage to the resistors. Despite high thermal stability, surface smoothness and low cost of glass substrate, the choice of PCB has been mainly suggested by the possibility to easily obtain the electronic contacts and to integrate the active electronic components on board. As a main drawback, the PCB sensor showed a higher power consumption due to its thermal inertia. In order to resolve this problem, a new version of the sensor was realised. The design changes suggested by experimental and simulation results have allowed to obtain a new more miniaturised sensor with a very low regeneration power consumption.

From a microfabrication point of view, the main drawback of this new sensor is related to the lower reproducibility compared to the glass sensor. In order to solve this problem, two different activities will be performed: (1) conditioning and calibration of the sensor by integrating active electronic components on board; (2) deposition of an intermediate layer of $\mathrm{SiO}_{2}$ devoted to assure:

- a good gold film adhesion at the PCB substrate,

- the same performances of the glass substrate in terms of reproducibility,

- a barrier to the thermal conduction which allows a further decrease of the thermal noise and power consumption.

\section{Acknowledgements}

The EMECAP project is financially supported by the European Commission DG XII.

\section{References}

[1] J.O. Nriagu, A global assessment of natural sources of atmospheric trace metal, Nature 338 (1989) 47-49.

[2] R. Ebinghaus, R.M. Tripathi, D. Wallschlager, S.E. Lindberg, in: R Ebinghaus, R.R. Turner, L.D. de Lacerda, O. Vasiliev, W. Salomons (Eds.), Mercury Contaminated Sites, Characterization, Risk assessment and Remediation, Springer-Verlag, Berlin, 1999, Chapter 1, pp. 3-50.

[3] J. Munthe, I. Wangberg, N. Pirrone, A. Iverfeld, R. Ferrara, R. Ebinghaus, R. Feng, K. Gerdfelt, G.J. Keeler, E. Lanzillotta, S.E. Lindberg, J. Lu, Y. Mamane, E. Nucaro, E. Prestbo, S. Schmolke, W.H. Schroder, J. Sommar, F. Sprovieri, R.K. Stevens, W. Stratton, G. Tuncel, A. Urba, Intercomparison of methods for sampling and analysis of atmospheric mercury species, Atmos. Environ. 35 (2001) 3007-3017.

[4] J.J. McNerney, P.R. Buseck, R.C. Hanson, Mercury detection by means of thin gold film, Science 178 (1972) 611-612.

[5] V. Bezak, M. Kedroand, A. Pevala, Longitudinal electrical conductivity of heterogeneous double-layer metallic films, Thin Solid Films 23 (3) (1974) 305-313.

[6] M. Levlin, E. Ikavalko, T. Laitinen, Adsorption of mercury on gold and silver surface, Fresenius J. Anal. Chem. 365 (7) (1999) 577-586.

[7] J.W. Geus, in: J.R. Anderson (Ed.), Chemisorption and Reactions on Metallic Films, vol. 1, Academic Press, London, 1971, p. 386.

[8] M.A. George, W.S. Glaunsinger, The electrical and structural properties of gold films and mercury covered gold films, Thin Solid Films 245 (1-2) (1994) 215-224.

\section{Biographies}

Barbara Mazzolai received her Laurea degree in biology (with Honours) from the University of Pisa, Italy in 1995. From 1994 to 1998, she worked at the Institute of Biophysics of the National Research Council on environmental topics and in particular, on mercury pollution. In 1998, she received her master's degree in eco-management and audit schemes (EMAS) organised by the Scuola Superiore Sant'Anna (SSSA), Pisa, Italy. From February 1999, she is a research assistant at Center for Research in Microengineering (CRIM) Laboratory, SSSA. Her main research interests are in the fields of microsystem technologies applied to environmental, agrofood and biomedical problems. She is currently working on several European and international projects focused on these fields.

Virgilio Mattoli received his Laurea degree in chemistry (with Honours) from the University of Pisa and the diploma in chemistry (with Honours) from the Scuola Normale Superiore of Pisa in 2000. He joined the CRIM Laboratory of Scuola Superiore Sant'Anna in 2002 as a PhD student with a research program focused on the control and integration of miniaturised devices for environmental and biomedical application. His main research interests include thin film sensor microfabrication, sensor conditioning and miniaturised acquisition system. He is currently involved in several research projects on these topics.

Vittoria Raffa received her degree in chemical engineering (with Honours) from the University of Pisa in 2001. She is a PhD student in microsystem engineering at the University 'Tor Vergata' in Rome. From 2002, she is working at CRIM Laboratory of the Scuola Superiore Sant'Anna in Pisa as research collaborator. Her research activity aims to develop microdevices and sensors for health-related applications (biomedical, environmental, food, etc.). She is currently involved in some European research projects on these topics. She is skilled in thin film 
properties and thin film deposition techniques and in microfabrication facilities.

Girolamo Tripoli received his Laurea degree in aeronautical engineering from the University of Palermo, Italy in 1999. He continued his studies at the University of Sheffield as a MPhil Student with a research program focused on the experimental stress analysis in weld steel plate. From October 2001, he is working as research assistant at CRIM Laboratory, SSSA. His main research interests include design of microfluidic component, computational analysis of microfluidic system, design of micromold.

Dino Accoto received his Laurea degree in mechanical engineering from the University of Pisa in 1998 and the diploma in engineering from the Scuola Superiore Sant'Anna in 1999. He joined the MiTech Lab of SSSA in 1999 as a PhD student with a research program focused on the design techniques and fabrication methods of microfluidic devices. During 2002, he was a visiting researcher at RPL Lab, Stanford University, where he worked on the design of efficient microvalves. From October 2002, he is a post-doctorate in biomedical engineering at CRIM Laboratory, SSSA. His main research interests include microfluidics, biomechanics of soft tissues and design of biomimetic microsystems. He is currently involved in several research projects on these topics.

Arianna Menciassi received her Laurea degree in physics (with Honours) from the University of Pisa in 1995. In the same year, she joined the MiTech Lab of the Scuola Superiore Sant'Anna in Pisa as a PhD student in bioengineering with a research program on the micromanipulation of mechanical and biological microobjects. In 1999, she received her $\mathrm{PhD}$ degree by discussing a thesis titled 'Microfabricated Grippers for Micromanipulation of Biological and Mechanical Objects'. She had a post-doctoral position at SSSA from May 1999 until April 2000. Then, she obtained a temporary position of assistant professor in bioengineering at SSSA. Her main research interests are in the fields of biomedical microrobotics, microfabrication technologies, micromechatronics and microsystem technologies. She is working on several European projects and international projects for the development of minimally invasive instrumentation for medical applications.

Paolo Dario (M'88-SM'01) received his Dr.Eng degree in mechanical engineering from the University of Pisa in 1977. Currently, he is a professor of biomedical robotics at the Scuola Superiore Sant'Anna, Pisa. $\mathrm{He}$ also established and teaches the course on mechatronics at the School of Engineering, University of Pisa. He has been a visiting professor at the Ecole Polytechnique Federale de Lausanne (EPFL), Lausanne, Switzerland, and at Waseda University, Tokyo, Japan. He is the director of the ARTS Laboratory and of the Center for Research in Microengineering Laboratory of SSSA, where he supervises a team of about 70 researchers and $\mathrm{PhD}$ students. His main research interests are in the fields of medical robotics, mechatronics and microengineering, and specifically in sensors and actuators for the above applications. He is the coordinator of many national and European projects, the editor of two books on the subject of robotics and the author of more than 200 scientific papers. He is a member of the Board of the International Foundation of Robotics Research. He is an associate editor of the IEEE Transactions on Robotics and Automation, a member of the Steering Committee of the Journal of Microelectromechanical Systems and a guest editor of the Special Issue on Medical Robotics of the IEEE Transactions on Robotics and Automation. He serves as president of the IEEE Robotics and Automation Society and as the cochairman of the Technical Committee on Medical Robotics of the same society. 